\title{
Study on Photocatalytic Degradation of Pollutants by Zinc Oxide Nanocomposites
}

\author{
Yuanyuan $\mathrm{Du}^{1 *}$, Xinjun $\mathrm{Li}^{1 *}$, Ying $\mathrm{Fu}^{1}$, Xing Gao ${ }^{1}$, Wen $\mathrm{He}^{1}$, Li Han ${ }^{1}$, Weijuan Gong ${ }^{1}$ and Pengwu Zheng ${ }^{2}$ \\ ${ }^{1}$ School of Chemical and Biological Engineering, Qilu Institute of Technology, Jinan Shandong. 250200. China \\ ${ }^{2}$ School of Pharmacy, Jiangxi Science and Technology Normal University, Nanchang Jiangxi. 330013. China \\ Yuanyuan $\mathrm{Du}^{1 *}$ and Xinjun $\mathrm{Li}^{1 *}$ contributed equally to this work.
}

\begin{abstract}
In recent years, the research of zinc oxide nanocomposites has attracted much attention.Due to the high probability of photonized carrier recombination of a single nanometer zinc oxide material,and the low utilization of sunlight,its application in the degradation of pollutants is limited.Compounding zinc oxide with other materials can solve this problem.In this paper,through the study of the photocatalytic properties of zinc oxide nanocomposites, the synthesis methods and photocatalytic properties of $\mathrm{TiO}_{2} / \mathrm{ZnO}$ and $\mathrm{ZnO} /$ graphene nanocomposites are reviewed.Nanocomposite photocatalytic degradation mechanism of pollutants and its application in the degradation of pollutants.
\end{abstract}

\section{Introduction}

In the contemporary era where the industrial level is rapidly developing, green and energy have become hard core issues in contemporary development. As a novel and effective green technology, photocatalysis has played a huge role in energy utilization. The current photocatalysts include $\mathrm{TiO}_{2}, \mathrm{ZnO}, \mathrm{WO}_{3}, \mathrm{ZnS}, \mathrm{CdS}, \mathrm{g}-\mathrm{C}_{3} \mathrm{~N}_{4}$, etc., which are the most widely used semiconductor photocatalysts, including oxides, Sulfides, new photocatalysts, etc. Among the semiconductor photocatalytic materials, $\mathrm{ZnO}$ is cheap, non-toxic, stable, hot spots, high photosensitivity, controllable crystal shape and excellent photocatalytic activity, and has attracted wide attention in the degradation of various pollutants ${ }^{[1]}$. However, because of the unique performance structure of $\mathrm{ZnO}$, it also encounters extremely severe problems in photocatalytic applications, because its electron-hole pair combination is more likely to be combined, resulting in its absorption and utilization of solar energy. The rate is relatively low. Since the discovery of $\mathrm{TiO}_{2}$ and graphene, researchers in this field have begun to discover a composite photocatalyst of $\mathrm{TiO}_{2}$, graphene and $\mathrm{ZnO}$, which can effectively improve the utilization efficiency of photocatalysis. This article mainly introduces the research progress of $\mathrm{TiO}_{2} / \mathrm{ZnO}$ and $\mathrm{ZnO} /$ graphene nanocomposites, their preparation methods, reaction mechanism and photocatalytic degradation of pollutants $^{[2]}$.

\section{Nano materials}

\subsection{Nanomaterials and their basic properties}

Nanomaterials refer to the substances stored in the nanometer. Its three-dimensional space size is at least onedimensional, composed of nanoparticles, and its size is between atoms, molecules and macroscopic systems . Nanotechnology refers to new materials that are directly processed on the nanometer depository, and are composed of one atom or molecule $\mathrm{e}^{[3]}$. Because nanomaterials are very small, the electronic activities inside them are not free, making them different from the electronic energy level structure of bulk solids, which specifically exhibits four characteristics.

(1) Surface effect

When the particle size is reduced to nanometer size, its specific surface area increases rapidly, the number of atoms on it increases, its surface energy increases, and the activity of the particle also increases.

(2) Small size effect

When the particle size is reduced to nanometer size, as its volume becomes smaller, the wavelength of the light source becomes shorter, the de Broglie wavelength becomes smaller, the size of the single magnetic domain becomes smaller, and the critical size condition of the particle lattice becomes worse. Out of an unstable state, there is a significant change in properties ${ }^{[4]}$.

(3) Quantum size effect

When the particle size is reduced to nanometer size, the continuous combination of electronic activities changes its electronic energy level structure, resulting in changes in physical properties.

(4) Macroscopic quantum tunneling effect

The ability of microscopic particles (electrons) to enter and penetrate an obstacle is called tunneling. The microscopic mechanism of the nanometer size will affect its macroscopic physical quantities, such as the magnetization and magnetic flux of microscopic particles. The macro-quantum tunneling effect shortens the length 
of time the optical disc stores information, and is bound to become a hard core in the field of microelectronics development, driving the further development of science and technology to miniaturization ${ }^{[5]}$.

\section{2 $\mathrm{ZnO}$ photocatalytic nanomaterials}

A prominent feature of nano-semiconductor materials is the photocatalytic feature. It can achieve high differentiation and degradation ability of pollutants under the condition of low energy consumption. This new technology material is called nano-semiconductor photocatalytic material, which plays a key role in the field of environmental sewage treatment. The principle of photocatalysis is that the electrons in the valence band of the semiconductors irradiated by sunlight are excited, and electron-hole pairs are formed in the conduction band ${ }^{[6]}$. The electrons have strong reductive properties and the holes have strong oxidizing properties. The compound on the surface of the catalyst undergoes a redox reaction with it upon stimulation .

$\mathrm{ZnO}$ photocatalytic reaction process: When $\mathrm{ZnO}$ is irradiated with high energy and strong light, electrons in the valence band are excited to the conduction band, and holes are generated in the valence band, and electron-hole pairs are generated inside the semiconductor. The holes oxidize the $\mathrm{OH}-$ and $\mathrm{H}_{2} \mathrm{O}$ molecules on the surface, and generate $\mathrm{OH} \bullet$. The generated $\mathrm{OH} \bullet$ can continue to oxidize adjacent organic substances, and $\mathrm{OH} \bullet$ can also oxidize organic substances in the liquid phase. Various organic substances undergo a series of oxidation processes. In the end, they all become $\mathrm{H}_{2} \mathrm{O}$ and $\mathrm{CO}_{2}$, and the degradation process of organic matter is completed ${ }^{[7]}$.

As a new type of photocatalyst, $\mathrm{ZnO}$ differentiates and degrades organic pollutants. The main problems of $\mathrm{ZnO}$ itself are: first, the probability of electron-hole pair binding is relatively high, resulting in low catalytic reaction efficiency; second, the utilization rate of solar energy is low. , Limiting its application. This article will describe the composite material formed by $\mathrm{TiO}_{2}$ or graphene combined with nano-zinc oxide, which prevents the combination of electron-hole pairs, increases the photocatalytic response range, and has excellent photocatalytic performance .

\section{Study on the photocatalytic performance of $\mathrm{TiO}_{2} / \mathrm{ZnO}$ nanocomposite}

In the fields of environmental beautification, pollution control, and biological sciences, photocatalysts play a very important role. By combining $\mathrm{TiO}_{2}$ and $\mathrm{ZnO}$ to increase the light absorption range, improve the absorption and utilization of solar energy, and prevent the combination of electron-hole pairs, In this way, the photocatalytic performance of Shanshan becomes better.

\section{1 $\mathrm{Nano}_{\mathrm{TiO}}$}

Nano- $\mathrm{TiO}_{2}$, green and environmentally friendly, is a heterogeneous semiconductor photocatalyst. It plays an important role in various application fields, such as qualified wastewater treatment and air purification quality standards. It is non-toxic and harmless, stable in chemical properties, low in energy efficiency, and can be used repeatedly for many times. Its high photocatalytic activity is a notable feature, and it is one of the most promising semiconductor materials ${ }^{[8]}$.

\subsubsection{Nano- $\mathrm{TiO}_{2}$ photocatalytic mechanism}

$\mathrm{TiO}_{2}$ is an n-type semiconductor material. When sunlight is irradiated, electrons in the valence band are excited and transition to the conduction band, and corresponding holes $\left(\mathrm{h}^{+}\right)$are generated in the valence band. Under the action of the electric field, the electrons and holes are separated and transferred to the particle surface ${ }^{[9]}$. A part of the electronhole pairs recombine, and the $\mathrm{O}_{2}, \mathrm{H}_{2} \mathrm{O}$ and $\mathrm{OH}^{-}$on its surface react with another part to form $\mathrm{HO} \cdot$, which then oxidizes various organic substances and is differentiated and degraded.

\subsubsection{Application of nano- $\mathrm{TiO}_{2}$ in the field of photocatalysis}

(1) Removal of wastewater pollutants

Wastewater mainly contains various pollutants such as phenols and hydrocarbons. Among them, the most toxic is heterocyclic compounds. Because of the complex composition of wastewater, it is difficult to separate the pollutants from the wastewater ${ }^{[10]}$.

(2) Air purification

Air purification has always been a key topic of green development today. At present, $\mathrm{TiO}_{2}$ occupies an important position in air purification, because it can effectively decompose air pollutants. $\mathrm{TiO}_{2}$ can be coated on the surface of various devices, can also be used in steel bars, and even directly used on pollution sources, which has played an important role in environmental governance.

(3) Sterilization, corrosion resistance and self-cleaning

$\mathrm{TiO}_{2}$ photocatalysis is used in all aspects of life, because it can not only decompose bacteria, but also sterilize and disinfect. Because of the difference in energy level, it can protect the metal surface from corrosion. It is excellent in hygiene and health. If it is applied to materials that are not easy to clean, it shows good self-cleaning ability. It also has a good function of sterilization and disinfection, strong practicability, and outstanding performance in antivirus of daily necessities ${ }^{[11]}$.

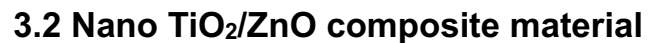

\subsubsection{Preparation of nano- $\mathrm{TiO}_{2 / \mathrm{ZnO}}$ composite materials}

At present, there are three main research methods for nano- $\mathrm{TiO}_{2} / \mathrm{ZnO}$ composite materials.

(1) Sol-gel method

When the organometallic compound or metal salt is processed, it will become a liquid colloidal substance after 
the hydrolysis reaction. After losing water, it will become a solid colloid. Apply it to the outermost layer of the substance ${ }^{[12]}$. Will form a hard protective film. We can also use other technologies to produce smaller particles. The main reason is that this method is simple to operate and low in cost. It can also be used for large-scale production of film products .

(2) Hydrothermal method

Using water as the reaction medium, heating the reaction kettle, insoluble or insoluble substances will become soluble under high temperature and high pressure conditions, and can be recrystallized. It is similar to the sol-gel method and also has the advantages of simple operation and low cost. Vendula et al. synthesized $\mathrm{TiO}_{2} / \mathrm{ZnS}$ by melting and degrading $\mathrm{TiOSO}_{4}$ and $\mathrm{ZnSO}_{4} \cdot 7 \mathrm{H}_{2} \mathrm{O}$, which was dried and then continuously calcined to generate a photoactive $\mathrm{TiO}_{2} / \mathrm{ZnO}$ nanocomposite. Small-molecule spherical substances constitute the material, and the more $\mathrm{TiO}_{2}$ content, the smaller the particles formed. Remi et al. used this method to obtain the $\mathrm{TiO}_{2} / \mathrm{ZnO}$ nanocomposite, which is uniformly dispersed and has a small average particle size ${ }^{[13]}$

\section{(3) Two-step method}

First, $\mathrm{TiO}_{2}$ nanostructures are generated, and the second hydrothermal method is used to synthesize $\mathrm{TiO}_{2} / \mathrm{ZnO}$ nanocomposites. Its advantages are: (1) The shape of the substance can be well controlled to obtain a satisfactory shape of the substance; (2) The obtained substance can realize the classification of nano-layers and has ultra-high performance.

\subsubsection{Photocatalytic degradation mechanism of nano- $\mathrm{TiO}_{2} / \mathrm{ZnO}$ composite}

When the composite is irradiated by solar energy, the electrons in the valence band will be stimulated and migrate to the conduction band, and the valence band will produce holes $(\mathrm{h}+): \mathrm{TiO}_{2} / \mathrm{ZnO}(\mathrm{hv}) \rightarrow \mathrm{TiO}_{2}-\mathrm{ZnO}$ Holes will oxidize various organics to generate $\mathrm{CO}_{2}$ and $\mathrm{H}_{2} \mathrm{O}$, which completes the degradation of organics. Because of the doping of other substances, the doped energy level will be generated, so that photons of small energy can excite the electrons and holes generated at this energy level, and the possibility of photons being absorbed and utilized is increased $^{[14]}$. The more doping, the greater the free length of carriers, the longer the survival time of electrons and holes, and the restriction of $\mathrm{e}^{-/} / \mathrm{h}^{+}$combination, which increases the possibility of solar energy absorption and utilization of the composite material, and the photocatalytic effect Excellent. It is concluded that composite materials have a much better catalytic effect than pure nano-materials as photocatalysts.

\subsubsection{Application of photocatalytic degradation of pollutants by nano- $\mathrm{TiO}_{2} / \mathrm{ZnO}$}

Under the conditions of $180{ }^{\circ} \mathrm{C}$ and $0.8 \mathrm{MPa}$, using lowtemperature and low-pressure hydrothermal method, nano- $\mathrm{TiO}_{2} / \mathrm{ZnO}$ composite materials can be synthesized. This material can absorb visible light well, of course, ultraviolet light is no exception, and it can fully differentiate and decompose acid red B And acid black 234 dye. It has one of the most significant advantages, it can directly use sunlight to optimize the space environment ${ }^{[15]}$.

According to the research of Fang Yanju et al., after the two substances are combined, the absorption spectrum will produce a red shift phenomenon, which significantly improves the ability of the composite film to oxidize glucose, which is higher than that of a single raw material. Zhang Hao et al. Pointed out that for the composite formed by the $\mathrm{Zn} / \mathrm{Ti}$ atomic ratio of $2: 1$, its photocatalytic performance is excellent, and the composite material has outstanding performance in degrading methylene blue, and its degradation ability is much higher than that of a single raw material.

Kostedt et al. studied the nanocomposite of the two substances, and made an objective judgment on the adsorption capacity of the red MX-5B dye and the photocatalytic oxidation capacity of the shoots. The study found that because these two raw materials have similar zeta potential and energy band energy, materials with a mixing ratio of almost $100 \% \mathrm{ZnO}$ or $\mathrm{TiO}_{2}$ exhibit excellent catalytic performance. $\mathrm{Rad}$ et al. believed that when the mass ratio of $\mathrm{ZnO}$ to $\mathrm{TiO}_{2}$ in the composite is $1: 1$, it has a more prominent performance effect on the degradation of red dyes. The larger the specific surface area of the composite, the more the composite is decomposed. The greater the possibility, on the contrary, the larger the particle size, the smaller the possibility of its differentiation and decomposition. Liu Yue et al. found that the composite has much higher catalytic activity than P25 when photocatalytic oxidation of NO. Under sunlight, when the atomic fraction of $\mathrm{Zn}^{2+}$ is $0.5 \%$, it prevents $\mathrm{OH}$ on the surface. The formation of nitroso groups makes NO easier to be oxidized, and the photocatalytic effect is the best.

SakaiA et al. found that the complex can be used to remove methyl mercaptan. $\mathrm{TiO}_{2}$ coated with $\mathrm{ZnO}$ improves the adsorption capacity of $\mathrm{TiO}_{2}$, especially for acidic substances. When the exposed part can absorb sunlight, photoions will be formed and $\mathrm{CH}_{3} \mathrm{SH}$ will be oxidized and decomposed. Ndrea.E et al. studied the $\mathrm{TiO}_{2} / \mathrm{ZnO}$ nanocomposite electrode and found that the more appropriate the amount of $\mathrm{ZnO}$ in the composite is controlled, the more breakthrough the photocatalysis problem is. Different materials have their own suitable composite ratio of $\mathrm{TiO}_{2} / \mathrm{ZnO}$. The photocatalytic reaction mechanism and the shape of the composite are also factors that influence the optimal composite ratio.

\section{Study on the photocatalytic performance of $\mathrm{ZnO/graphene}$ nanocomposite}

$\mathrm{ZnO}$ /graphene nanocomposite material helps to separate electron-hole pairs effectively. Because of its large specific surface area, it plays an important role in the diffusion and mass transfer of pollutants and free radicals. The hole of $\mathrm{ZnO}$ is on the high-energy conduction band. The electrons can be directly transferred to graphene, 
which greatly improves its photocatalytic performance under ultraviolet or visible light.

\subsection{Graphene}

\subsubsection{The structure and properties of graphene}

Graphene, a hexagonal single-layer carbon sheet, is the basic unit of carbon materials . It is formed by the way of hybridization of carbon atoms and presents a hexagonal crystal structure. C-C is connected by a $\sigma$ bond, and a $120^{\circ}$ bond is formed between $\mathrm{C}-\mathrm{C}$, and the distance between $\mathrm{C}$ $\mathrm{C}$ is about $0.142 \mathrm{~nm}$. Its single layer thickness is only 0.35 $\mathrm{nm}$, which is extremely light and thin.

Graphene is a semi-metallic material with no band gap . Its ultraviolet penetration effect is very good. Each layer of graphene has an absorption rate of only $2.3 \%$ for ultraviolet and visible light. It also has extremely high transparency in the near and mid-infrared bands, and its mechanical strength has always been the first. With the same excellent thermal properties, BalandinAA et al. measured that single-layer graphene has a higher thermal conductivity of $5.2 \mathrm{~kW}$. (m.K)-1 than carbon materials such as diamond and carbon nanotubes. The super large specific surface area makes it possess extremely strong adsorption capacity.

\subsubsection{The production method of graphene}

1. Peeling method, including mechanical peeling method [39] and chemical peeling method.

2. Reduction and oxidation method. The graphite is oxidized to obtain graphene oxide $(\mathrm{GO})$, and $\mathrm{GO}$ is reduced by chemical reduction method, electrochemical reduction method, thermal expansion reduction method and hydrogen reduction method.

\subsection{ZnO/graphene nanocomposite}

$\mathrm{ZnO}$ nanoparticles are uniformly dispersed and bound to graphene, which can increase its specific surface area and charge separation more easily.

\subsubsection{Preparation of ZnO/graphene nanocomposite}

Using the hydrothermal method, the $\mathrm{ZnO} / \mathrm{GO}$ composite material obtained by Pokharel $\mathrm{P}$ et al. is petal-shaped, which improves the possibility of combining photoelectrons and holes and improves the photocatalytic performance of the composite material. Using the uniform precipitation method, He Guangyu et al. prepared $\mathrm{ZnO} / \mathrm{GO}$ composite materials. Because of the electron transfer effect between the two kinds of nanoparticles, the combination of photogenerated electron-hole pairs is limited, and the visible light catalytic performance is greatly improved. increase.

Using the one-step method, Sun Hongqi et al. produced a $\mathrm{ZnO} / \mathrm{CTAB} / \mathrm{GO}$ composite material with low band gap energy, fast electron transfer, and strong adsorption of organic dyes. Hou Zongwei et al. obtained a zinc oxide nanorod/graphene composite photocatalyst, and used a vacuum-assisted pressure induction method to input the reaction solution into the expanded graphite room to synthesize the material.

Using the microwave-assisted synthesis method, Liu Xinjuan et al. synthesized $\mathrm{ZnO} / \mathrm{GO}$ composite materials with zinc nitrate and graphite oxide as reactants, which increased the absorption range of ultraviolet light, reduced the neutralization effect, and increased the electron transfer efficiency.

Using the one-pot method, Zou Changwu et al. produced $\mathrm{ZnO} /$ graphene nanocomposite microspheres, which accelerated the speed of electron transfer and had a good effect on the differentiation and decomposition of methyl blue under ultraviolet light.

Using the auxiliary method of ionic liquids, Zhang Kai et al. developed a $\mathrm{ZnO} /$ graphene composite material with a $\mathrm{p} / \mathrm{n}$ heterojunction, which improved the possibility of photoelectrons and holes binding, and improved the ability to adsorb pollutants. improve.

\subsubsection{The photocatalytic degradation mechanism of $\mathrm{ZnO/graphene} \mathrm{nanocomposite}$}

Light absorption, generation and separation of electronholes, electron-hole transfer, and oxidation-reduction reactions are the key steps for the material to differentiate and decompose pollutants. Mainly manifested in : First, because graphene is mixed, the material has a larger surface area, and the diffusion and mass transfer of pollutants and free radicals is further improved; second, the high-energy conduction band of $\mathrm{ZnO}$ is $-4.05 \mathrm{eV}$, Its low-energy valence band full of electrons is $-7.25 \mathrm{eV}$, while graphene is $-4.42 \mathrm{eV}$. The electrons on the conduction band of $\mathrm{ZnO}$ can be directly transferred to graphene, and the photocatalytic performance of the composite material is improved. Third, graphene has a strong e-capturing ability, which can effectively separate e- and make $\mathrm{O}_{2}$ react with it to produce $\mathrm{O}_{2}^{-}$, and $\mathrm{OH}-$ captures the separated $\mathrm{h}^{+}$to produce $\mathrm{OH}$, which makes it possible to combine electrons and holes. The performance decreases and the photocatalytic efficiency increases. Therefore, composite materials have a much better catalytic effect than pure nano-materials as photocatalysts.

\subsubsection{Application of ZnO-graphene nanocomposite material for photocatalytic degradation of pollutants}




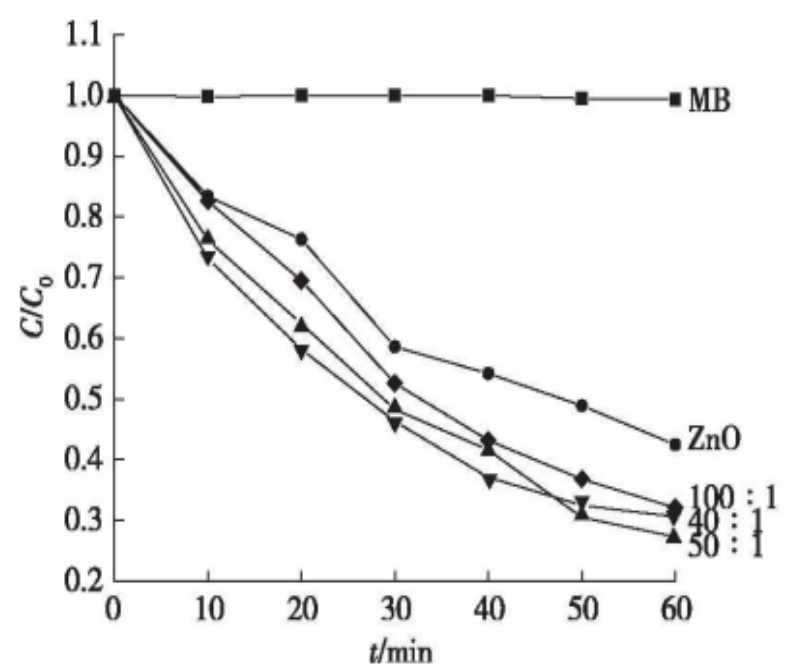

Figure 1 Degradation of methylene blue solution (without any catalyst (MB); pure $\mathrm{ZnO} ; \mathrm{ZnO}$ /graphene mass ratio 100:1, 50:1, 40:1) under ultraviolet light

Figure 1 shows the changes in the degradation rate of methylene blue over time when pure $\mathrm{ZnO}$ and $\mathrm{ZnO}$ /graphene are selected as catalysts. MB was used as a blank control, which did not add any catalyst, which can be observed under the irradiation of ultraviolet light. At this time, the nature of the methylene blue solution is very stable, and the concentration of the solution is basically unchanged. It can also be observed that the photocatalytic performance of all $\mathrm{ZnO} /$ graphene samples is better than pure $\mathrm{ZnO}$. This is because in the composite material where zinc oxide is in the graphene sheet structure, they have very close band gaps, and the electrons on the valence band of zinc oxide can directly migrate into graphene. The energy required for this process is not high, so It makes the transmission and transfer of photo-generated electrons more convenient, and the photocatalytic performance is improved. When the mass ratio of graphene in the composite material changes from $100: 1$ to $40: 1$, its photocatalytic activity will show a peak and then decrease. This peak value is when the mass ratio is 50:1, the material exhibits the strongest activity When the photocatalytic time reaches $60 \mathrm{~min}$, using the formula $\mu=\left(1-\mathrm{C} / \mathrm{C}_{0}\right) \times 100 \%$, the efficiency of photocatalytic degradation of methylene blue solution at this time is calculated to be higher than $70 \%$, and at this time, the mass ratio is 100 The photocatalytic decomposition efficiency of : 1 is $67.9 \%$, and the photocatalytic decomposition efficiency of 40:1 by mass is $69.4 \%$. The results indicate that the highest efficiency of photocatalytic degradation is that the $\mathrm{ZnO}$ /graphene composite material has a mass ratio of When the ratio of 50:1 appears, the efficiency of photocatalytic degradation of methylene blue solution is slightly lower than that of other ratios. Under the irradiation of ultraviolet light, the mass ratio of $\mathrm{ZnO} /$ graphene changes from 100:1 to 50:1, the electron migration speed becomes faster, the possibility of electron-hole pair combination is lower, and the photocatalytic performance of the material is higher; The graphene content continues to increase to $40: 1$, but the photocatalytic performance decreases. The reason should be: when the mass ratio of graphene in the composite material continues to increase, after the mass ratio of
$\mathrm{ZnO} /$ graphene exceeds 50:1, it will inevitably increase The thickness of graphene results in poor transparency, resulting in a decrease in the intensity of light that can reach $\mathrm{ZnO}$, which in turn reduces the number of photogenerated electron-hole pairs in $\mathrm{ZnO}$, which will reduce the photocatalytic performance. Therefore, the best quality ratio of $\mathrm{ZnO} /$ graphene should be around 50:1.

\section{Summary}

For the nano-ZnO raw material, because it has a small band gap width, it can be used in the photocatalytic decomposition of pollutants, but its shortcomings limit the improvement of its performance. Due to the low valence band potential and poor light stability, the raw material is light. The effect of catalytic differentiation and decomposition of pollutants is not ideal. In view of the advantages and disadvantages of a single raw material, nanocomposite $\mathrm{TiO}_{2}$ and $\mathrm{ZnO}$, and prepare a $\mathrm{TiO}_{2} / \mathrm{ZnO}$ nanocomposite system. The greater the coupling between the nanoparticles, the greater the possibility of solar energy absorption and utilization, and the organic matter is differentiated The faster the rate of decomposition will make it more and more important in the differentiation and decomposition of pollutants. Nano-composite $\mathrm{ZnO}$ and graphene, combine $\mathrm{ZnO}$ particles on graphene through different experimental methods and synthesize composite materials. As a green advanced composite, it plays a very important role in photocatalytic applications, especially in Differentiating and decomposing pollutant dyes has both theoretical value and practical significance. As far as the current research status is concerned, the removal and mechanism of new refractory pollutants are not perfect enough, and we still need to continue to work hard. We also believe that with the continuous development of nanotechnology, people are concerned about the photocatalytic degradation of pollutants by zinc oxide nanocomposites. Research will also go to the next level.

\section{Reference}

1. Zhang Zhonghai, Yuan Yuan, Fang Yanju, et al. Preparation of photocatalytic nano- $\mathrm{ZnO} / \mathrm{TiO}_{2}$ film and application for determination of Chemical oxygen demand[J]. Talanta, 2007, 73: 523.

2. Chen Da, Zhang Hao, $\mathrm{Hu}$ Song,et al.Preparation and enhanced photoelectro-Chemical performance of coupled bicomponent $\mathrm{ZnO}-\mathrm{TiO}_{2}$ nanocomposites[J]. J Phys Chem C, 2008, 112: 117.

3. William L, Kostedt I V, Adel A Ismail, et al. Impact of heat treatment and composition of $\mathrm{ZnO}-\mathrm{TiO}_{2}$ nanoparticles for photocatalytic oxidation of an azo dye[J]. Ind Eng Chem Res, 2008, 47: 1483.

4. Asl S K, Sadrnezhaad K, Rad M Kianpoor. Photocatalytic decolorization of red dye in aqueous ZnO- $\mathrm{TiO}_{2}$ suspensions[J]. Smart Mater, 2008, 55-57: 577.

5. Wang Haiqiang, Wu Zhongbiao, Liu Yue, et al. The characterization of $\mathrm{ZnO}$-anatase-rutile threecomponent semiconductor and enhanced 
photocatalytic activity of nitrogen oxides[J]. J Mol Catal A, Chem, 2008, 287: 176.

6. Sakai A, Kanehata M, Ogawa T, et al. Improvement of methyl mercaptan removal by $\mathrm{ZnO}$-coated $\mathrm{TiO}_{2}[\mathrm{~J}]$. Trans Mater Res Soc Japan, 2007, 32(4): 1079.

7. Indrea E, Dreve S, Silipas D T, et al. Semiconductor photoelectrodes for solar of splitting water[J]. J Optoelectron Adv Mater, 2008, 10(9): 2213.

8. Zhu Y, Murali S, Cai W, et al. Graphene and graphene oxide: synthesis, properties, and applications[J]. Advanced Materials, 2010, 22(35): 3906-3924.

9. King A, Johnson G, Engelberg D, et al. Observations of intergranular stress corrosion cracking in a grainmapped polycrystal[J]. Science, 2008, 321(5887): 382-385.

10. Nair R R, Blake P, Grigorenko A N, et al. Fine structure constant defines visual transparency of graphene[J]. Science, 2008, 320(5881): 1308.

11. King A, Johnson G, Engelberg D, et al. Observations of intergranular stress corrosion cracking in a grainmapped polycrystal[J]. Science, 2008, 321(5887): 382-385.

12. Balandin A A, Ghosh S,Bao W, et al. Superior thermal conductivity of single-

layer graphene[J]. Nano Letters, 2008, 8(3): 902-907.

13. Novoselov K S, Jiang Z, Zhang Y, et al. Roomtemperature quantum hall effect in graphene[J]. Science, 2007, 315(5817): 1379.

14. Geim A K, Morozov S V, et al. Electric fieldeffect in atomi[J]. Science, 2004, 306(5696): 666-669.

15. Si Y, Samulski E T. Synthesis of water soluble graphene[J]. Nano Letters, 2008, 8(6): 1679-1682. 